\title{
Lipid apheresis: oxidative stress, rheology, and vasodilatation
}

\author{
K.-P. Mellwig • E. Pulawski $\cdot$ D. Horstkotte $\cdot$ \\ F. van Buuren
}

Published online: 10 March 2012

(C) The Author(s) 2012. This article is published with open access at Springerlink.com.

\begin{abstract}
In the treatment of homozygous and therapyresistant hypercholesterolemia, lipid apheresis enables not only low density lipoprotein (LDL) cholesterol to be lowered by approximately $60 \%$, but also oxidative stress factors to be influenced and adhesion molecules reduced. This was investigated in a group of 12 patients using the heparin-induced extracorporeal LDL precipitation (H.E.L.P.) procedure.

A significant lowering of LDL cholesterol and fibrinogen leads to an improvement in rheology and endothelial function, detectable and measurable within approximately $20 \mathrm{~h}$ by assessing minimum coronary resistance using positron emission tomography (PET) performed in 35 patients. This effect is detectable even after the first lipid apheresis session (H.E.L.P. procedure), documented in 12 patients.

Lipid apheresis appears to be the most effective procedure in the treatment of elevated lipoprotein(a) [Lp(a)]. A chosen group of nine patients with selective elevated $L p(a)$ illustrated both the influence on endothelial dysfunction, in the shape of sharply increased minimum coronary resistance, and the reduction through lipid apheresis, indicating that $L p(a)$ seems to exert a similar effect on the vascular wall and vascular function as LDL cholesterol.
\end{abstract}

Keywords Lipid apheresis - Hypercholesterolemia · Lipoprotein(a)

Lipid apheresis is a tried and tested therapeutic procedure for the treatment of familial homozygous and therapy-re-

K.-P. Mellwig $(\bowtie) \cdot$ E. Pulawski $\cdot$ D. Horstkotte $\cdot$ F. van Buuren Department of Cardiology, Heart and Diabetes Center North Rhine-Westphalia, Ruhr University of Bochum,

Bad Oeynhausen, Germany

e-mail: kpmellwig@hdz-nrw.de sistant hypercholesterolemia in conjunction with coronary artery disease. Targeted lowering of LDL cholesterol below 100 or $80 \mathrm{mg} / \mathrm{dl}$ as secondary prevention in high-risk patients [1] is not realizable in some patients despite the use of highly potent lipid-lowering drugs (statins). In such cases, alternative therapeutic procedures are required, especially in conjunction with advancing coronary artery disease.

Here lipid apheresis represents a therapeutic option. Over the past 25 years, various techniques [2] have become available and found their way into hospitals and clinical practices. The most widespread are LDL adsorption by dextran sulfate (DAS) bound to cellulose and heparin-induced extracorporeal LDL precipitation (H.E.L.P.) [3, 4].

In addition to lowering LDL cholesterol, the dominant factor in the pathogenesis of atherosclerosis, additional factors such as oxidative stress, adhesion molecules, and fibrinogen, which maintain atherosclerosis, influence endothelium in its function as a perfusion-relevant organ and reduced vasodilatation capacity.

\section{Oxidative stress and hypercholesterolemia}

Severe hypercholesterolemia is associated with an increased concentration of lipid peroxidation products in the serum. An extension of the half-life of LDL particles in the circulatory system is the result, caused by missing and/or defective LDL receptors or by impaired LDL internalization. This leads to an increased susceptibility of the LDL particles to oxidation. The consequence is a formation of minimally modified LDL and oxidized LDL (OxLDL) [5].

Markers of oxidative lipid damage are OxLDL, isoprostanes, and malondialdehyde. Just the contact of leukocytes with artificial surfaces during extracorporeal circulation can trigger the release of chemokines and cytokines as well as 
the discharge of free radicals (respiratory burst) [6], disturbing the balance of antioxidants and prooxidants.

Patients and methods

We investigated the influence of a single H.E.L.P. apheresis session on the plasma concentration of parameters of lipid peroxidation before (pre-) as well as after (post1) apheresis, as well as 1 week later, immediately prior to the next apheresis treatment (post2) in 12 patients (aged $57 \pm 10$ years, six women) with heterozygous familial hypercholesterolemia and known CAD who were participating in the chronic weekly H.E.L.P. apheresis program at our hospital. The plasma concentrations of OxLDL, copper/zinc superoxiddismutase $(\mathrm{Cu} / \mathrm{ZnSOD})$, and 8-isoprostane-PGF2a were determined using enzyme-linked immunosorbent assay (ELISA) measurements, of malondialdehyde (MDA) using high-performance liquid chromatography (HPLC), and the antioxidative serum capacity ( $\mathrm{ImAnOx})$ using photometry.

Results

Immediately prior to the H.E.L.P. treatment, the following mean values were measured in the patients: total cholesterol $255 \pm 52 \mathrm{mg} / \mathrm{dl}$, LDL cholesterol $164 \pm 44 \mathrm{mg} / \mathrm{dl}$, HDL cholesterol $60 \pm 11 \mathrm{mg} / \mathrm{dl}$, and triglyceride $135 \pm 70 \mathrm{mg} / \mathrm{dl}$.

The single H.E.L.P. treatment reduced the total cholesterol by $53 \%$, the LDL cholesterol by $54 \%$, and the OxLDL by $47 \%$. The serum concentrations of MDA, the stable degradation product of lipid peroxidation, were significantly lowered, as was the antioxidative serum capacity (ImAnOx). In contrast, the plasma concentration of cellular $\mathrm{Cu} / \mathrm{ZnSOD}$ underwent no change as a result of the H.E.L.P. apheresis (Table 1).

\section{Conclusion}

Following the H.E.L.P. treatment, in most patients an increase in 8-isoprostane-PGF2a concentration in the serum could be observed. It is possible that the 8-isoprostanePGF2a predominantly originate from the cell membranes of activated platelets. The other direct parameters of the lipid peroxidation OxLDL and MDA were, however, significantly reduced as a result of H.E.L.P. apheresis.

The prooxidative processes are quantitatively inferior to antioxidative effects right in the foreground, so that in the H.E.L.P. apheresis the sum of antioxidative effects is far greater.

\section{Additional effects of lipid apheresis: adhesion molecules}

The adhesion of circulating leukocytes to the endothelial cells and the following transendothelial migration represent a decisive step in the initiation of atherosclerosis [7]. During this process, adhesion molecules such as ICAM-1, VCAM1, and P-selectin assume a key role. Patients with hyperlipidemia display a higher plasma concentration of adhesion molecules. It was possible to show that through the inhibition of ICAM-1 and P-selectin the vascular inflammation process could be almost completely suppressed.

Patients and methods

In 12 patients with advanced CAD and insufficiently controlled (through drugs/diet) hypercholesterolemia, we investigated the effect of single-session LDL apheresis on the adhesion molecules ICAM-1, VCAM-1, and P-selectin, as well as changes in the intervals between treatments. The plasma concentrations of sVCAM-1, sICAM-1, and P-selectin were each determined by ELISA measurement before (pre) and after (post1) the LDL apheresis, as well as immediately prior to the next LDL apheresis (post2). In addition, the plasma concentrations of the adhesion molecules were determined directly in front of and behind the precipitation filter.

\section{Results}

In each case, the reduction in the plasma concentration of the adhesion molecules behind the precipitation filter was $70 \%$ for P-selectin, $62 \%$ for sVCAM- 1 , and $56 \%$ for sICAM-1. In terms of percentage, the reduction in the serum concentrations of the adhesion moleculwas $26.0 \pm 13.2 \%$ for

Table 1 Mean values measured in the patients immediately prior to the H.E.L.P. treatment

\begin{tabular}{lllllll} 
& Pre & Post1 & Pre vs. post1 & Post2 & Pre vs. post2 \\
\hline OxLDL $(\mathrm{mU} / \mathrm{l})$ & $11.4 \pm 6$ & $6.25 \pm 3$ & $\mathrm{p}<0.01$ & $10 \pm 3$ & n.s. & n.s. \\
MDA $(\mu \mathrm{mol} / \mathrm{l})$ & $1.27 \pm 0.3$ & $0.9 \pm 0.14$ & $\mathrm{p}<0.01$ & $1.25 \pm 02$ & n.s. \\
ImAnOx $(\mu \mathrm{mol} / \mathrm{l})$ & $317 \pm 35$ & $287 \pm 46$ & $\mathrm{p}=0.03$ & $312 \pm 29$ & n.s. \\
$\mathrm{Cu} / \mathrm{ZnSOD}(\mathrm{ng} / \mathrm{ml})$ & $75 \pm 36$ & $71 \pm 27$ & n.s. & $64 \pm 20$ & n.s. \\
8-Isoprostane-PGF2a $(\mathrm{pg} / \mathrm{ml})$ & $28 \pm 11.3$ & $39.7 \pm 24$ & $\mathrm{p}<0.05$ & $30.4 \pm 12$ & \\
\hline
\end{tabular}

$O x L D L$ oxidized LDL; $M D A$ malondialdehyde; ImAnOx antioxidative serum capacity; n.s. not significant 
P-selectin, $29.1 \pm 9.0 \%$ for sVCAM-1, and $15.5 \pm 8.4 \%$ for sICAM-1 and was proportional to the molecular weight. Within 1 week the effect was no longer visible.

\section{Conclusion}

The significant reduction in ICAM-1, VCAM-1, and P-selectin can most probably be attributed to a filter effect. The additional impact of the lipid apheresis, in particular of the H.E.L.P. procedure [8], on the reduction in adhesion molecules can possibly impact the progression of atherosclerosis by influencing the leukocyte adhesion tendency.

\section{Rheology and vasodilatation}

In patients with coronary artery disease, an improvement in endothelial function and endothelium-dependent coronary vasodilatation capacity can be achieved through intensive lowering of cholesterol levels $[9,10]$. This can be explained by the interactions of LDL cholesterol (LDL) and nitrogen monoxide (NO), which synthesizes in the endothelial cell and is released from there [11]. In an oxidized state, high LDL concentrations reduce the bioavailability of NO and alter the myocardial vascular tone by inhibiting the endothelium-dependent vasodilatation conveyed by receptors and flow. Reducing fibrinogen, an independent cardiovascular risk factor, leads to an improvement in plasma viscosity and a reduction in the aggregation tendency of the erythrocytes [12]. Despite a share of just $4 \%$ in the total protein, due to its molecular size $(341,000 \mathrm{Da})$ and length $(700 \mathrm{~nm})$, fibrinogen is responsible for $20-25 \%$ of the plasma viscosity [13]. It is unclear how fast the LDL-associated limitation of coronary vasodilatation capacity can be normalized or decisively improved in conjunction with endothelial dysfunction.

\section{Patients and methods}

Forty-seven patients ( 12 women, 35 men, aged $50 \pm 9$ years) with hypercholesterolemia and angiographically documented coronary artery disease were examined. Thirtyfive patients ( 11 women, 24 men, aged $50 \pm 8$ years) were participating in the chronic lipid apheresis program with regular treatments taking place once a week (Group A). The mean preceding period of treatment was 53.1 months (5-98 months). Twelve patients ( 1 woman, 11 men, aged $47 \pm 9$ years) were undergoing lipid apheresis for the first time (Group B). The lipid apheresis was performed using the H.E.L.P. procedure.

Quantification of myocardial perfusion was performed using positron emission tomography (PET) $[9,16]$. The tracer substance used was ${ }^{13} \mathrm{~N}$-ammonia from our own cyclotron production (Cyclone 18/9).

In order to evaluate coronary vasodilatation capacity, the following parameters were determined: myocardial blood flow at rest (MBFbasal), maximum myocardial blood flow under dipyridamole (MBFD), and minimum coronary resistance as a reciprocal measure of coronary conductance calculated from the quotients of mean arterial pressure and MBFD.

In accordance with the study protocol passed by the ethics commission, the first laboratory diagnosis was performed on the day of treatment prior to the PET examination (PRE). Immediately after that, lipid apheresis was performed. At the end of the treatment the effectivity was documented by a second laboratory diagnosis (POST 1). The next day the patients in both groups underwent a second PET examination as well as laboratory diagnosis, after an interval of 18-20 h (POST 2).

\section{Results}

Through the lipid apheresis, a significant lowering of total cholesterol, LDL cholesterol, and fibrinogen could be achieved in Group A and B (see Table 2). Immediately before the follow-up PET examination in Group A, the reduction in total cholesterol was $41.3 \%$, and in both LDL cholesterol and fibrinogen $45.8 \%$.

Immediately before the follow-up PET examination in Group B, the reduction in total cholesterol was $43.2 \%$, in LDL cholesterol 54.6\%, and in fibrinogen 51.8\%.

The changes in viscosity in Group A (baseline: $1.28 \pm 0.10 \mathrm{mPa} \mathrm{s}$; at the time of the follow-up PET examination $1.18 \pm 0.08 \mathrm{mPa} \mathrm{s}$, corresponding to a reduction by $7.8 \%$ ) were significant $(\mathrm{p}<0.01)$.

In Group B, the baseline viscosity was $1.29 \pm 0.08 \mathrm{mPa}$. At the time of the follow-up PET examination it was $1,19 \pm 0.07 \mathrm{mPa} \mathrm{s}$, corresponding to a reduction by $7.6 \%$. This was also significant $(\mathrm{p}<0.0075)$.

The minimum coronary resistance (MCR) dropped in Group A from $0.56 \pm 0.20$ to $0.44 \pm 0.17 \mathrm{mmHg} \min 100 \mathrm{~g} / \mathrm{ml}$ $(\mathrm{p}<0.0001)$. In Group B, the minimum coronary resistance

Table 2 Lowering of total cholesterol, LDL cholesterol, and fibrinogen through lipid apheresis in Group A and B

\begin{tabular}{|c|c|c|c|c|c|c|c|c|c|}
\hline \multirow[t]{2}{*}{ Group } & \multicolumn{3}{|c|}{ Cholesterol (mg/dl) } & \multicolumn{3}{|c|}{ LDL cholesterol (mg/dl) } & \multicolumn{3}{|c|}{ Fibrinogen (mg/dl) } \\
\hline & $\overline{\text { PRE }}$ & POST 1 & POST 2 & $\overline{\mathrm{PRE}}$ & POST 1 & POST 2 & $\overline{\text { PRE }}$ & POST 1 & POST 2 \\
\hline A & $251 \pm 58$ & $120 \pm 22$ & $145 \pm 28$ & $175 \pm 50$ & $60 \pm 21$ & $77 \pm 25$ & $287 \pm 75$ & $102 \pm 29$ & $150 \pm 52$ \\
\hline B & $256 \pm 48$ & $126 \pm 27$ & $145 \pm 30$ & $187 \pm 45$ & $75 \pm 27$ & $85 \pm 30$ & $348 \pm 65$ & $126 \pm 38$ & $168 \pm 45$ \\
\hline
\end{tabular}


dropped from $0.61 \pm 0.23$ to $0.53 \pm 0.19 \mathrm{mmHg} \min 100 \mathrm{~g} /$ $\mathrm{ml}(\mathrm{p}<0.01)$

\section{Conclusion}

The significant lowering of LDL cholesterol leads to a significant lowering of minimum coronary resistance, the most sensitive parameter for evaluating vasodilatation capacity. It should be emphasized that these effects are detectable within $18-20 \mathrm{~h}$ and are most probably attributable to a considerable improvement in NO bioavailability [14].

A change in stenosis morphology is not to be expected within this short examination period.

With regard to Group B, the fact that a significant improvement in coronary vasodilatation capacity could be achieved after just one H.E.L.P. apheresis session is of particular clinical significance [15].

\section{Lipoprotein(a)}

Lipoprotein(a) $[\mathrm{Lp}(\mathrm{a})]$ is a fat-protein molecular complex and consists of a low density lipoprotein (LDL) particle which is bound to a glycoprotein similar to plasminogen, apolipoprotein(a) [16]. The structural affinity of $L p(a)$ to LDL and plasminogen indicates, in pathophysiological terms, that in the atherosclerosis process it has a proatherogenous effect like LDL. In addition, it reinforces its effects and promotes thrombotic events due to a structure homologous to plasminogen.

Since the early 1960s, Lp(a) has been discussed as a significant and independent risk factor for cardiovascular diseases [17].

It could be proven that rising $\mathrm{Lp}$ (a) plasma levels are linked to an increase in the risk of infarction, while extreme increases in Lp(a) above $85 \mathrm{mg} / \mathrm{dl}$ increase the risk of myocardial infarction three to fourfold [18].

Patients and methods

In accordance with the above-mentioned protocol, nine patients (aged $53 \pm 10$ years, one woman) with a normal LDL cholesterol concentration $(95 \pm 13 \mathrm{mg} / \mathrm{dl})$ and a high $\mathrm{Lp}(\mathrm{a})$ concentration $(147 \pm 67 \mathrm{mg} / \mathrm{dl})$ underwent lipid apheresis (H.E.L.P.) together with a PET examination.

Results

LDL cholesterol dropped from 95 to 34 or $39 \mathrm{mg} / \mathrm{dl}$ prior to the second PET examination (reduction: 60.2/55.5\%), fibrinogen from 318 to 124 or $164 \mathrm{mg} / \mathrm{dl}$ (reduction: $61.1 / 48.5 \%$ ), viscosity from 1.24 to 1.06 or $1.11 \mathrm{mPa}$ s. The
$\mathrm{Lp}$ (a) concentration dropped from 147 to 50 or $60 \mathrm{mg} / \mathrm{dl}$ (reduction: 66/59.2\%).

As a result, MCR could be reduced from 0.52 to $0.42 \mathrm{mmHg} \min 100 \mathrm{~g} / \mathrm{ml}(\mathrm{p}<0.0001)$.

Conclusion

In conjunction with severely increased $\mathrm{Lp}$ (a) concentration and normal LDL concentration, a high MCR is particularly conspicuous. A significant improvement in perfusion was achieved through the sharp decrease brought about by H.E.L.P. apheresis. In all probability, $\mathrm{Lp}$ (a) in a high concentration influences coronary perfusion and leads to impaired endothelial function in the same way as high-LDL concentrations.

\section{Summary}

Lipid apheresis, in particular the H.E.L.P. procedure, not only leads to a significant reduction in LDL cholesterol and $\mathrm{Lp}(\mathrm{a})$; impact on further parameters such as oxidative stress factors and adhesion molecules, favorably influences both the pathogenesis and the progression of atherosclerosis. The key statement here, however, is that the functionality of the coronary system, detectable by perfusion or vasodilatation capacity, improves. This ultimately explains clinical improvement in patients undergoing this treatment without any essential change to coronary morphology.

Acknowledgments This article is part of a supplement sponsored by an unrestricted educational grant from B. Braun and Fresenius Medical Care.

Conflict of Interest The authors declare that there is no conflict of interest.

Open Access This article is distributed under the terms of the Creative Commons Attribution License which permits any use, distribution, and reproduction in any medium, provided the original author(s) and the source are credited.

\section{References}

1. Graham I et al (2007) European guidelines on cardiovascular disease prevention in clinical practice: full text. Fourth Joint Task Force of the European Society of Cardiology and other societies on cardiovascular disease prevention in clinical practice (constituted by representatives of nine societies and by invited experts). Eur J Cardiovasc Prev Rehabil 14(Suppl 2):1-113

2. Julius U et al (2008) Comparison of different LDL apheresis methods. Expert Rev Cardiovasc Ther 6(5):629-639

3. Wieland H, Seidel D (1983) A simple specific method for precipitation of low density lipoproteins. J Lipid Res 24(7):904-909 
4. Schuff-Werner P et al (1994) The HELP-LDL-apheresis multicentre study, an angiographically assessed trial on the role of LDLapheresis in the secondary prevention of coronary heart disease. II. Final evaluation of the effect of regular treatment on LDL-cholesterol plasma concentrations and the course of coronary heart disease. The HELP-Study Group. Heparin-induced extra-corporeal LDL-precipitation. Eur J Clin Invest 24(11):724-732

5. Mashima R, Witting PK, Stocker R (2001) Oxidants and antioxidants in atherosclerosis. Curr Opin Lipidol 12(4):411-418

6. Jackson P et al (1995) Effect of hemodialysis on total antioxidant capacity and serum antioxidants in patients with chronic renal failure. Clin Chem 41(8 Pt 1):1135-1138

7. Ridker PM (1998) Intercellular adhesion molecule (ICAM-1) and the risks of developing atherosclerotic disease. Eur Heart $\mathbf{J}$ 19(8):1119-1121

8. Pulawski E et al (2002) Influence of single low-density lipoprotein apheresis on the adhesion molecules soluble vascular cellular adhesion molecule-1, soluble intercellular adhesion molecule-1, and p-selectin. Ther Apher 6(3):229-233

9. Gould KL et al (1994) Short-term cholesterol lowering decreases size and severity of perfusion abnormalities by positron emission tomography after dipyridamole in patients with coronary artery disease. A potential noninvasive marker of healing coronary endothelium. Circulation 89(4):1530-1538

10. Hutchins GD, Schwaiger M, Wolfe E (1993) Positron emission tomography to quantitate myocardial perfusion. Am J Card Imaging 7(4):283-293
11. Liao JK (1994) Inhibition of Gi proteins by low density lipoprotein attenuates bradykinin-stimulated release of endothelial-derived nitric oxide. J Biol Chem 269(17):12987-12992

12. Schuff-Werner P, Seidel D (1989) Improved haemorheology associated with a reduction in plasma fibrinogen and LDL in patients being treated by heparin-induced extracorporal LDL precipitation (HELP). Eur J Clin Invest 19:30-37

13. Lowe GD et al (1980) Relation between extent of coronary artery disease and blood viscosity. Br Med J 280(6215):673-674

14. Mellwig KP et al (1998) Improvement of coronary vasodilatation capacity through single LDL apheresis. Atherosclerosis 139(1):173-178

15. Mellwig KP et al (2006) Improved coronary vasodilatatory capacity by h.e.L.p. Apheresis: comparing initial and chronic treatment. Ther Apher Dial 10(6):510-517

16. Kostner KM, Kostner GM (2002) Lipoprotein(a): still an enigma? Curr Opin Lipidol 13(4):391-396

17. Berg K, Mohr J (1963) Genetics of the Lp System. Acta Genet Stat Med 13:349-360

18. Kamstrup PR et al (2008) Extreme lipoprotein(a) levels and risk of myocardial infarction in the general population: the Copenhagen City Heart Study. Circulation 117(2):176-184 